\title{
Case Report and Review of the Literature
}

\section{Forgotten Double J Ureteral Stent for 10 years: A Case Report with Review of Literature}

\author{
Hamdy Aboutale B $^{1, *}$ \\ ${ }^{1}$ Professor of urology, Menoufia University hospitals, Menoufia, Egypt \\ ${ }^{2}$ Consultant Urologist, Burjeel hospital, Abu Dhabi, UAE
}

\begin{tabular}{l} 
A R T I C L E I N F O \\
\hline Article history: \\
Received: 21 October, 2020 \\
Accepted: 31 October, 2020 \\
Published: 11 November, 2020 \\
\hline Keywords: \\
Forgotten double J stent \\
double J stent \\
ureteric calculus \\
bladder calculus
\end{tabular}

\begin{abstract}
A B S T R A C T
Background: Ureteral stents are a crucial part of urologic surgeries. Complications of Double J stenting (DJS) include migration, fragmentation, and encrustation. In addition, forgotten stents with encrustations and stone formations are difficult to remove.

Case Presentation: We report the case of a stent "forgotten" for 10 years, which migrated downward into the proximal ureter, concomitant with multiple ureteral stones and bladder calculus. Whole encrustation of the stent was observed. The "forgotten" stent was positioned 10 years ago during right ureteral stone treatment in the Philippines. In the end, the patient underwent laser cystolithotripsy, stone elimination, ureteroscopic laser lithotripsy and stent removal. The final X-ray post-intervention revealed the absence of any stone fragment or residual DJS fragment.

Conclusion: We report the longest period of forgotten DJS with maximum stone burden in the urinary system. To avoid this situation, patients should be educated regarding complications if the stent is not removed within a short period. Moreover, a computerized registry of stent placement can be used to alert the urologist when the stent ought to be removed.
\end{abstract}

\section{Background}

Since 1978, urologists began using double $\mathrm{J}$ stents (DJS) in their practice. These stents are normally used in the treatment of obstructive anuria, ureteral reconstructive surgery, before extracorporeal shockwave lithotripsy (SWL), and obstructive uropathy. Over the last decade, stent design and material have been upgraded to reduce complications. However, serious complications such as migration, fragmentation, encrustation, and stone formation may occur, especially when stents are left in respective locations for long periods [1]. In addition, a forgotten stent may be complex and challenging at the time of removal [2]. Herein, we report a case of a forgotten DJS for 10 years that was complicated due to massive stone burden.

\section{Case Presentation}

A 49-year-old male patient from the Philippines presented to our outpatient department with intermittent right flank ache and burning micturition from the past one month. Abdominal and pelvic ultrasonography (US-KUB) revealed moderate hydronephrosis at the right side and bladder calculus. X-ray KUB (Figure 1) revealed a DJS forgotten in the interior that was inserted during treatment of ureteric stone in the Philippines 10 years ago. The whole stent was covered with a thick layer of encrustation with more than one stone formation. The stent migrated to the proximal ureter. A couple of radio-opaque shadows were observed: $27 \mathrm{~mm} \times 10 \mathrm{~mm}$ calculus inside the proximal ureter, a $32 \mathrm{~mm} \times 11 \mathrm{~mm}$ calculus at the level of sacroiliac joint, and $22 \mathrm{~mm} \times 12$ $\mathrm{mm}$ calculus at the level of the pelvic ureter. A $42 \mathrm{~mm} \times 22 \mathrm{~mm}$ calculus

${ }^{*}$ Correspondence to: Hamdy Aboutaleb, M.D., Ph.D., Urology, Professor of urology, Urology Department, Menoufia University Hospitals, Menoufia, Egypt; Burjeel Hospital, Abu Dhabi, UAE; Tel: 009710505323804; Fax:00971025085555; E-mail: hamdyabotaleb@yahoo.com 
was visible in the urinary bladder. The total stone burden in the ureter was $81 \mathrm{~mm}$, and the stone bladder was $42 \mathrm{~mm}$ (Overall stone burden 123 $\mathrm{mm})$. These findings were confirmed using CT KUB scan.

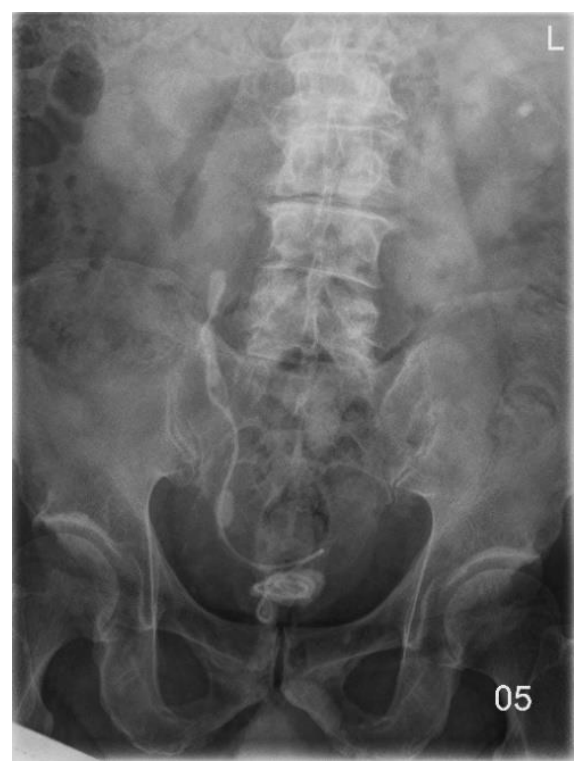

Figure 1: X-ray kidney-ureter-bladder revealed a forgotten double $\mathrm{J}$ stent for 10 years with bladder calculus formed in the lower coil and multiple stones in the ureter with upper coil in the proximal ureter.

Preoperative laboratory examinations revealed a total leucocyte count of 12,000 per cu mm. Urine microscopy revealed plenty of pus cells and RBCs. The levels of serum creatinine $(132 \mathrm{mmol} / \mathrm{L})$, uric acid $(484$ $\mathrm{mmol} / \mathrm{L})$, and serum calcium $(2.9 \mathrm{mmol} / \mathrm{L})$ were found to be elevated. The direction of the ailment and diagnosis were explained to the affected person. The patient's fitness for surgical treatment was assessed by using an anesthesiologist. Treatment selection was based on clinical presentation and diagnostic imaging findings. In this case, we decided to endorse a combination of transurethral cystolithotripsy with Holmium YAG laser for the bladder calculus and semirigid/flexible ureteroscopy with Holmium YAG laser lithotripsy for ureteral stones plus encrusted stent.

\section{Operative Procedure}

\section{First Session}

Briefly, the patient received prophylactic parenteral antibiotics (Ceftriaxone) and general anaesthesia with a laryngeal mask. Briefly, Cystoscope 22 Fr was inserted for diagnostic cystourethroscopy and used for cystolithotripsy. The bladder calculus (Figure 2) was fragmented by cystourethroscopy and Holmium YAG laser Lithotripsy, and the gravels were eliminated using Ellik evacuator. Then, semi-rigid ureteroscope 6-7.5 $\mathrm{F}$ was used for diagnostic/therapeutic ureteroscopy (URS), a 0.64-mm straight tip Terumo guidewire was smoothly placed in the kidney, and a 0.89 -mm straight tip Roadrunner guidewire (Cook Urological Inc., USA) was inserted. The lower ureter stones and encrustations over the lower part of the stent were removed by Semirigid Ureteroscopic Holmium YAG Laser lithotripsy (Richard Wolf Medical Instruments Corp., USA).

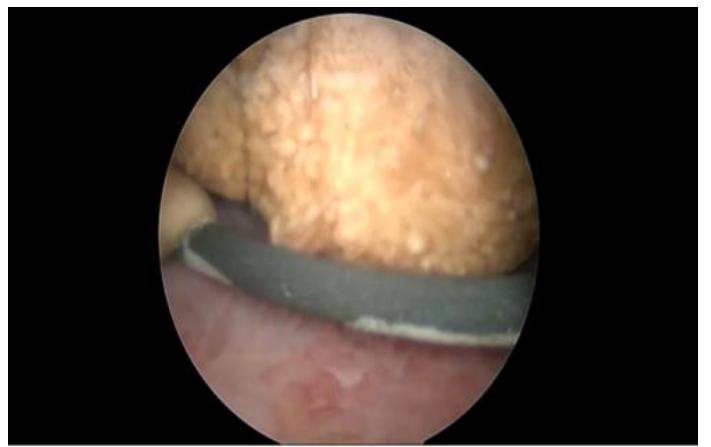

Figure 2: Endoscopic view of bladder calculus is formed in the lower coil of the forgotten double $\mathrm{J}$ stent for 10 years.

\section{Second Session}

This session pertained to the management of proximal ureteric stones (Figure 3). In brief, with the help of a semi-rigid ureteroscope, a 0.64$\mathrm{mm}$ straight tip Terumo guidewire was smoothly placed inside the kidney, and a 0.89-mm straight tip Roadrunner guidewire was inserted. The flexible ureteroscope (Richard Wolf Medical Instruments Corp., USA) has outer and tip diameters of $9.9 \mathrm{~F}$ and $6 \mathrm{~F}$, respectively. This instrument has identical upwards and downwards deflection of $270^{\circ}$. The Ho: YAG laser was inserted (200-272 $\mu \mathrm{m}$ fiber, Mega pulse stone laser, Richard Wolf Medical Instruments Corp.) for fragmentation. The laser apparatus settings were adjusted to provide $200-4000 \mathrm{~mJ}$ with a pulse frequency of 3-25 Hz. After complete fragmentation, the process was stopped when only very small stone fragments $(2 \mathrm{~mm})$ were visible, preventing the need for basket stone retrieval. The laser fiber was then withdrawn. When the stone was fragmented into significant fragments (>3 mm), the fragments were removed with zero-tip N Gage basket (Cook Urological Inc., USA). The middle and proximal ureteral stones were cleared, and encrustation over the middle and upper part of the stent was eliminated within the second consultation.

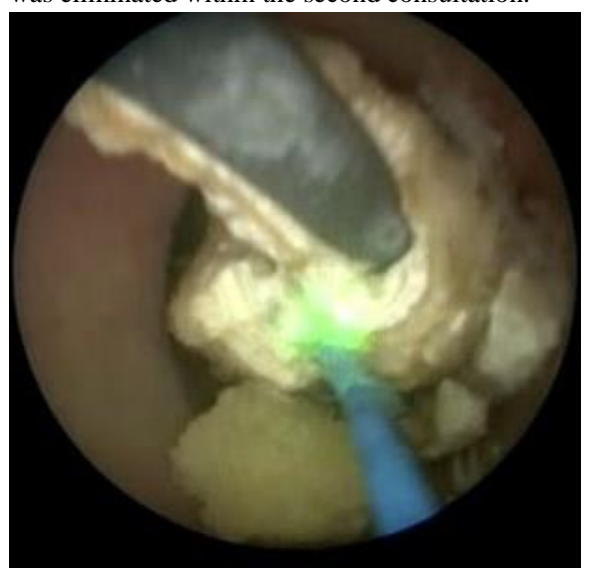

Figure 3: Endoscopic view of the forgotten double $\mathrm{J}$ stent with thick layers of encrustation around the stent during removal by holmium YAG laser.

The patient successfully underwent ureteroscopic removal of the upper ureteral stone and the stent fragments within the second consultation (Figure 4). The final plain X-ray demonstrated new DJS within the right accumulation system with stone fragments or residual DJS fragment diagnosed. After the procedure, the patient was asymptomatic and currently remains so. Postoperative stone evaluation revealed the stone 
composition to include calcium oxalates monohydrates, calcium oxalates dihydrates, and calcium phosphates. Stent swab culture revealed infection by Burkholderia spp.

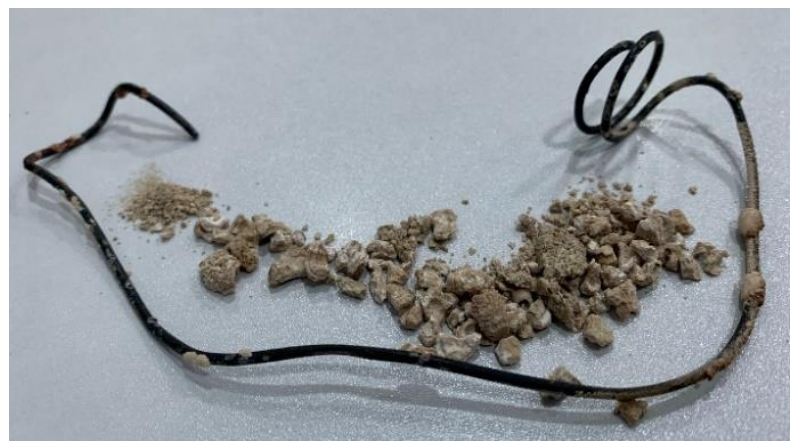

Figure 4: Picture shows the removed forgotten double $\mathrm{J}$ stent with peeled outer layer and multiple stone gravels.

\section{Discussion}

Ureteral stents are frequently utilized in urologic practices. Stent composition and design have undergone many improvements but are still associated with morbidity in approximately $80 \%$ of cases [3]. Aboutaleb et al., reported DJS complications in 170 patients, with postoperative lower urinary tract irritative symptoms in the form of frequency and urgency in $63.5 \%$ patients, gross hematuria in $15.9 \%$ patients, and colic in $13.5 \%$ patients [1]. Upward migration of the stents was observed in $1.2 \%$ patients, while downward migration was observed in $5.9 \%$ patients. Encrustations were observed in $11.8 \%$ patients.

Monga et al., reported 22 patients with "forgotten" stents for more than 6 months [4]. Complications were reported as migration by $40.9 \%$ patients, calcified stents by $68 \%$ patients, fragmented stents by $45.5 \%$, and fragmented with calcification by $13.6 \%$. Aboutaleb et al., reported histopathological changes inside the urinary bladder because of the temporary presence of DJS [5]. They observed that these reactions extended and became extreme as the stent duration increased. In our previous report, we discovered the inflammatory response became extra excessive and obvious with Brunn's nests at 4 to 6 weeks [5]. Cystitis cystica, Brunn's nests, and cystitis glandularis were observed frequently in patients who had stents lasting more than 12 weeks. It is clear from the study that, when the period of stenting increased, the reaction changed from acute stage to chronic stage, especially after three months.

To our knowledge, this current study reports the longest duration of a "forgotten" stent, which was inserted 10 years ago, with the highest stone burden and neglected DJS reported in the literature (Figure 2). Moreover, the "forgotten" stent migrated down to the proximal ureter, inflicting obstruction and moderate hydronephrosis. Stent migration, fragmentation, and encrustation are the most common complications associated with forgotten DJS. In our report, we found that the patient had hypercalcemia and hyperuricemia with alkaline urine. Encrustation of DJS is often associated with alkaline urine and urinary tract infection (UTI), and struvite and calcium phosphate deposits. Andriole et al., reported a $10 \%$ incidence of stent encrustation in patients without history of stone disease [6]. Thus, in patients with a history of stones, encrustation is anticipated to be higher [7].

\section{Management of Complicated DJS}

This calls for coordinated use of the latest modern endourologic lithotripsy techniques. Combined surgical procedures or staged procedures are also required, especially in complicated cases. Shock wave lithotripsy (SWL), ureteroscopic laser lithotripsy, and percutaneous nephrolithotomy (PCNL) are the most frequently used techniques [8]. Due to the location of the stent in our patient, it was easy to retrieve the stent completely using a retrograde ureteroscopic procedure. Consequently, the PCNL technique was not required. We finally removed the stent completely using this method in staged procedures. LeRoy et al. also reported PCNL retrieval of complicated ureteral stents [9]. They recommended this approach over the retrograde method for patients with ureteral strictures, significant fragmentation, or calcified stent remnants in the renal pelvis.

\section{Prevention of Stent Complications}

Numerous measures are recommended to prevent DJS complications. Optimum length and placement of stents should minimize stent migration. Timely cystoscopic replacement needs to be done to minimize calcification and fragmentation when the stent is indicated for a long time. Earlier replacement may be required in patients with stones or during pregnancy. Prophylactic antibiotic therapy may decrease infected stone encrustation. In our case, the "forgotten" stent was because the patient was not aware about its presence and traveled to a different country for 10 years. To prevent this situation, patients should be reminded of the presence of an internal stent that could lead to problems if left inside for more than 3 months. Moreover, a computerized registry of stent placement may be used to alert the urologist when the stent must be removed.

\section{Conclusion}

We report the longest duration of forgotten DJS with highest stone burden in urinary system. To prevent this situation, patients should be educated for stent complications if not removed within a short period. Moreover, a computerized registry of stent placement may be used to alert the urologist when the stent must be removed.

\section{Acknowledgements}

Not applicable.

\section{Author Contributions}

HA wrote the manuscript, performed the operation, and managed the patient's perioperative course. HA gave the final approval of this manuscript. HA read and approved the final manuscript.

\section{Funding}

Not applicable.

\section{Ethical Approval and Consent}

All procedures performed in this study were in accordance with the ethical standards of the Institution and/or National Research Committee 
and with the 1964 Declaration of Helsinki and its later amendments or comparable ethical standards.

\section{Consent for Publication}

Written informed consent was obtained from the patient for publication of this case report and any accompanying images.

\section{Competing Interests}

None.

\author{
Abbreviations \\ DJS: Double J Stent \\ SWL: Extracorporeal Shock-Wave Lithotripsy \\ Holmium YAG Laser: Holmium: Yttrium-Aluminum-Garnet Laser \\ URS: Ureteroscopy
}

\section{REFERENCES}

1. Aboutaleb HA, Ali TA, Gawish M, Omar MK (2019) Fluoroscopy-free double-J stent placement through ureteroscope working channel postuncomplicated ureteroscopic laser lithotripsy: A novel technique. Urol Ann 11: 39-45. [Crossref]
2. Chen CK, Li CC, Ke HL, Chou YH, Huang CH et al. (2003) Double J Stent Forgotten for 7 Years: A Case Report. Kaohsiung J Med Sci 19: 84-87. [Crossref]

3. Joshi HB, Okeke A, Newns N, Keeley FX Jr, Timoney AG (2002) Characterization of urinary symptoms in patients with ureteral stents. Urology 59: 511-516. [Crossref]

4. Monga M, Klein E, Castañeda Zúñiga WR, Thomas R (1995) The forgotten indwelling ureteral stent: a urological dilemma. J Urol 153: 1817-1819. [Crossref]

5. Aboutaleb H, Gawish M (2017) Correlation of Bladder Histopathologic Changes Due to Double-J Stenting to the Period of Stenting: A Preliminary Study. J Endourol 31: 705-710. [Crossref]

6. Andriole GL, Bettmann MA, Garnick MB, Richie JP (1984) Indwelling Double-J ureteral stents for temporary and permanent urinary drainage: experience with 87 patients. J Urol 131: 239-241. [Crossref]

7. Spirnak JP, Resnick MI (1985) Stone formation as a complication of indwelling ureteral stents: a report of 5 cases. J Urol 134: 349-351. [Crossref]

8. Lupu AN, Fuchs GJ, Chaussy CG (1986) Calcification of ureteral stent treated by extracorporeal shock wave lithotripsy. J Urol 136: 12971298. [Crossref]

9. LeRoy AJ, Williams HJ Jr, Segura JW, Patterson DE, Benson Jr RC (1986) Indwelling ureteral stents: percutaneous management of complications. Radiology 158: 219-222. [Crossref] 